\title{
La gestion des déchets radioactifs à vie longue : confiés à l'argile ou bien gérés par la société?
}

\author{
Georges Mercadal ${ }^{*}$,a \\ Ancien vice-président de la Commission nationale du débat public (2002-2007), 92260 Fontenay-aux-Roses, France.
}

Reçu le 6 juin 2019 / Accepté le 3 juillet 2019

\begin{abstract}
Résumé - La révision du Plan national de gestion des déchets et matières radioactives (PNGMDR) exige l'organisation d'un débat public par la Commission nationale du débat public (CNDP). C'est l'occasion de rappeler les résultats du débat public de 2005-2006, notamment en ce qui concerne le stockage géologique profond des déchets radioactifs ultimes à vie longue. Une alternative au projet CIGEO avait été proposée lors de ce débat: «Confiés à l'argile vs. gérés par la société ». Cette proposition alternative n'avait pas été retenue à l'époque pour différentes raisons. Cet article a pour but de revisiter cette alternative et sa pertinence encore aujourd'hui dans le présent débat national.
\end{abstract}

Mots clés : déchets radioactifs / CNDP / confiance / sociotechnique

\begin{abstract}
The management of long life radioactive waste: entrusted to the clay vs. managed by the society? The revision of the French national plan of management of radioactive waste and nuclear matters (PNGMDR) requires the organization of a public debate by the national commission of public debate (CNDP). This is the occasion to recall the results of the public debate of 2005-2006, especially regarding the geological disposal of the ultimate long half-life radioactive waste. An alternative to the CIGEO project was proposed during that debate: "Entrusted to the clay $v s$. managed by the society". This alternate proposition had not been retained at that time for many reasons. The goal of this article is to revisit this alternative and its pertinence in the present national debate.
\end{abstract}

Keywords: radioactive waste / CNDP / trust / sociotechnical

\section{Introduction}

La Commission nationale du débat public (CNDP) a lancé le 4 avril 2018, conformément à la loi et à la demande du ministre de la Transition écologique et solidaire, un débat public pour l'élaboration de la cinquième édition triennale du Plan national de gestion des déchets et matières radioactives (PNGMDR). En effet, la CNDP considère qu'il convient d'associer le public à l'identification des priorités d'action des pouvoirs publics dans le domaine de la gestion des matières et déchets radioactifs dont les enjeux en matière de sûreté, de sécurité et de santé publique sont majeurs (CNDP, 2018). En bref, il s'agit de mettre en place une grande concertation avec la population au sujet de la gestion des déchets radioactifs en France (CNDP, 2019).

\footnotetext{
*Auteur de correspondance : georges.mercadal@free.fr

${ }^{a}$ Garant de concertations particulières (2002-2012).
}

L'animation de ce débat public a été confiée par la CNDP à une Commission particulière du débat public (CPDP) avec un calendrier de débat entre le 17 avril et le 15 septembre 2019 dont les rencontres portent sur de nombreux sujets comme le retraitement ou non des combustibles nucléaires usés, la gestion des déchets issus du démantèlement, la saturation des capacités d'entreposage, la gestion des déchets radioactifs ultimes ou les impacts sanitaires ou environnementaux...

Parmi toutes ces thématiques, celle du stockage géologique profond des déchets radioactifs ultimes à vie longue (HA-VL) est un sujet majeur et il s'agit en particulier de faire le point sur les avancées du projet de Centre industriel de stockage géologique (CIGEO) mis en œuvre par l'Agence nationale pour la gestion des déchets radioactifs (Andra). Dans ce domaine précis, l'Institut de radioprotection et de sûreté nucléaire (IRSN) a pour sa part rendu public le 15 mai 2019 les deux expertises qui lui avaient été demandées par la CNDP et qui portent sur les alternatives au stockage profond (IRSN, 2019a) et sur les possibilités d'entreposage à sec (IRSN, 2019b). 
Les questions soulevées ne sont pas nouvelles. En effet, «Confiés à l'argile vs. gérés par la société » fut l'alternative lancée en 2005-2006 par le premier débat public organisé par la CNDP sur la gestion de ces déchets radioactifs à vie longue.

Cette controverse était toujours très présente en 2018 dans l'esprit des opposants à CIGEO. En témoignent deux conférences/débats, organisées à Bar-le-Duc par l'association Jean Goguel et l'association des élus opposés à CIGEO et portant respectivement sur "La recherche d'une solution définitive pour ces déchets radioactifs à vie longue» et sur «Pourquoi la conclusion du débat de 2005-2006, qui avait ouvert cette alternative, n'avait pas été suivie par les pouvoirs publics ». Quinze ans après, cela interroge. Les contributions de 2005-2006 pourraient-elles être encore utiles aujourd'hui? Telles quelles ou du moins dans leur essence car le contexte a changé?

Les décisions récentes de la CNDP donnent une actualité à cette interrogation sur les déchets radioactifs à vie longue. Car, en programmant dans le débat PNGMDR, après les contacts qu'elle a eus avec les opposants, une réunion publique à Barle-Duc le 20 juin 2019 intitulée : «Les alternatives à CIGEO», la CNDP crée la réouverture du dialogue presque au point où il a été laissé en 2006. L'échec du débat de 2013 est réparé. De sorte que le regard se porte sur ce que sera l'attitude des responsables, pris entre des propositions d'alternatives, notamment la transmutation par laser, et la loi de 2016 qui légitime définitivement CIGEO (Legifrance, 2006b). Que vont faire ces responsables? Jouer la légitimité de la loi? Ou essaieront-ils de faire eux aussi une ouverture pour gagner la confiance?

C'est cette actualité qui a inspiré mon intervention du 20 mai 2019 lors d'un séminaire de préparation de l'IRSN au débat PNGMDR, sous l'égide de son Bureau de l'ouverture à la société (le BOS) : «Le débat de 2005-2006 peut-il suggérer aux ingénieurs du nucléaire, en 2019, une voie d'ouverture? Et s'il y en a une, a-t-elle des chances de convaincre les acteurs?». C'est tout l'objet de cet article de faire le point sur cette alternative «Confiés à l'argile $v s$. gérés par la société » au sujet des déchets radioactifs à vie longue qui posent bien entendu une question fondamentale de protection des personnes contre les rayonnements ionisants qu'ils émettent, dans la ligne éditoriale de ce journal (Bourguignon et al., 2016).

\section{Une stratégie pour la confiance, la leçon de 2005-2006}

C'est bien le recul du temps qui permet de mieux comprendre aujourd'hui qu'à l'époque les choix réalisés. Car il est significatif que sur les quatre contributions de ce débat exposées au Comité de l'énergie atomique en février 2006 par l'auteur, seule la quatrième soit restée dans les mémoires.

La première portait sur le temps : on n'est pas pressés disait le débat, et il faut de 15 à 20 ans de recherche encore sur l'enfouissement pour être sûrs. La loi de 2006 a donné 10 ans (Legifrance, 2006b).

La seconde avait trait au périmètre : il y a une kyrielle de déchets disait le débat. La loi de 2006 également sur la transparence nucléaire (Legifrance, 2006a) a donné force législative au PNGMDR, recommandé une concertation permanente pour l'élaborer, et instauré l'Autorité de sûreté nucléaire $(\mathrm{ASN})$ pour que la sûreté nucléaire soit dite par une Autorité administrative indépendante. Le débat d'aujourd'hui sur le PNGMDR en est la conséquence directe.

Le troisième thème émergeant du débat, concernant les attachements du public à son territoire et le drame de sa dépopulation, a certes retenu l'intérêt du gouvernement, mais sans accepter les moyens institutionnels suggérés par le débat.

Enfin la quatrième contribution du débat de 2006 était de proposer une stratégie capable de créer la confiance entre les responsables et la société civile. «Puisqu'on a 15 ans, étudions en parallèle aux recherches du laboratoire de Bures, une solution d'entreposage pérennisé ». Et sur ce point la réponse du Parlement fut un Non, assorti de la condition que le stockage soit réversible, condition qu'un rapport du gouvernement et un nouveau débat public devaient vérifier.

Le mouvement écologique et les associations locales ont reçu ce Non à grande déception. «Le débat n'a servi à rien » est vite devenu leur antienne. Cette déception s'est transformée en frustration lorsque la CNDP a refusé, en 2013, d'ouvrir le débat demandé par le parlement sur la réversibilité et une nouvelle discussion sur la stratégie de 2006. Et au printemps 2018, c'est devant un public que la loi de 2006 (Legifrance, 2006b) mettait en colère qu'il a fallu expliquer pourquoi le quatrième point de 2006 était rejeté avec constance par les responsables.

Cette dynamique se comprend lorsqu'on rappelle comment cette proposition a été construite. Elle est partie d'une sensibilité clairement exprimée par le public en faveur de la surveillance contre l'oubli, et une réactualisation de l'entreposage de longue durée. Ceci s'est transformé en entreposage pérennisé lorsque le directeur de l'énergie du CEA a répondu que « oui, on peut dans un entrepôt détecter les colis défectueux» et «oui on peut les réparer». C'était faire confiance à la sociologie plutôt qu'à la géologie. Puis à Dunkerque lors de l'avant dernière séance, Benjamin Dessus a formulé ce que le compte rendu de cette séance (CNDP, 2006) a retenu comme la quatrième contribution du débat: «puisqu'on doit poursuivre les recherches sur l'enfouissement, on a du temps, étudions en temps masqué et en parallèle l'entreposage pérennisé ».

Que s'est-il joué derrière ces mots? Pourquoi cette proposition de stratégie a-t-elle eu une telle force? Des déclarations on ne peut plus solennelles, faites sur le moment, nous mettent sur la piste. C'est d'abord à la séance conclusive de Lyon en 2006, où le directeur de l'énergie, Dominique Maillard dit: «On a deux solutions, c'est mieux qu'une. Nos successeurs choisiront». Et plus explicitement encore, lors du Comité à l'énergie atomique de février 2006 le président d'EDF, Pierre Gadonneix, qui, s'adressant aux trois ministres qui président la séance, déclare: «Quand je parle avec mes homologues étrangers, il est clair que le principal actif d'EDF est la confiance que la population française accorde au nucléaire. Si pour maintenir cette confiance il faut effectuer les deux études que propose le débat public, Messieurs les ministres, n'hésitez pas une seconde ».

La force de cette stratégie tient à ce qu'elle était ordonnée à la confiance entre ingénieurs et société civile. Tout le confirme aujourd'hui au vu des leçons apprises dans tous les débats publics. Ce que j'ai appelé la critique sociotechnique des projets construits dans les débats 
(Mercadal, 2011) se concentre sur trois axes : la défiance, les peurs, les attachements. Ce sont ces sentiments collectifs que provoquent, et nourrissent les analyses techniques des experts opposants. La défiance est de loin le premier d'entre eux, provoquée par les défauts du projet, mais plus encore par les défauts de la procédure et surtout par l'attitude des responsables. On pourrait remplir des pages de citations extraites des Verbatim pour le prouver. Mais est-ce nécessaire? Nous sommes dans une société de la défiance envers les institutions, ceux qui les dirigent, ceux qui savent ou croient savoir. Nous en voyons les effets tous les jours et à tout propos.

Au point que dans la trilogie défiance-peurs-attachements, les trois axes qui définissent l'espace dans lequel se déploie la critique sociotechnique, il faut faire une place à part à la confiance. Celle-ci est devenue la condition sans laquelle la décision publique est inacceptable. Pierre Rosanvallon le conceptualise en distinguant légitimité et confiance. L'action publique n'est acceptée que si elle réunit les deux qualités, comme l'illustre la période de grâce dont jouissent les présidents de la république. Mais celle-ci, nous le voyons amplement en ce moment, ne dure qu'un moment. La règle est la dissociation entre la légitimité conférée par le vote et lui seul, et la confiance générée par les comportements, la manière dont l'élu et ses services s'y prennent pour préparer la décision. L'affaire de Notre-Dame-des-Landes le rappelle : la légitimité sans la confiance, ça ne marche plus. Un référendum voulu par le président de la République pour trancher la question, un référendum gagné, quoi de plus légitime, et un projet abandonné à la suite de la perte de confiance au long des années (Caussade et al., 2016)!

\section{Pour la confiance aujourd'hui : une stratégie adaptée aux nouvelles données sociétales}

Stockage géologique versus entreposage pérennisé, c'était donc aussi, et plus profondément, légitimité d'une solution définitive, élaborée par l'ingénierie interne de l'autorité, versus confiance grâce à une stratégie ouverte de comparaison entre des voies différentes, créant possiblement des fertilisations croisées entre elles. Cette identification éclaire le choix qui se posera aux responsables après la réunion publique de Bar-le-Duc du 19 juin 2019 sur les alternatives au stockage réversible. Soit s'adosser à la légitimité de la loi et poursuivre CIGEO comme prévu. Soit opter pour donner la priorité à la confiance, et alors le débat de 2005 fournit une stratégie: sans ralentir le cours de CIGEO, lancer en temps masqué des recherches sur des voies alternatives qui pourront déboucher: soit sur une solution de remplacement soit sur des améliorations du stockage géologique.

Cette stratégie doit être adaptée à ce que l'on peut percevoir de la société aujourd'hui. Car plus personne ne défend l'entreposage pérennisé pendant des millénaires. Par contre l'échelle d'un entreposage pluriséculaire de deux cents ans semble bien une échelle à laquelle tout le monde admet le « faire confiance à la société »: le projet actuel puisque c'est le temps où le stockage est géré «comme un entrepôt» pour assurer la réversibilité, et les opposants puisqu'ils le prônent en attendant la solution de la transmutation par laser. Enfin, on voit que la réversibilité d'un stockage géologique suscite de nouvelles peurs, sans réellement répondre à l'idée que les profanes se font de la réversibilité.

Ces constats sociétaux amènent à conclure qu'adapter aux réalités d'aujourd'hui la stratégie de 2006, ce n'est plus la géologie contre la sociologie, le stockage réversible contre l'entreposage pérennisé. Mais nous pensons que ce peut-être la sociologie et la géologie, l'entreposage actif de quelques siècles puis le stockage scellé pour les millénaires. Des colis gérés dans un entrepôt pendant la période de réversibilité pour éviter les complications, et les peurs nouvelles, introduites par la réversibilité en contexte minier. Des colis qui auraient pendant ce temps refroidi, et seraient stockés dans l'argile sans risque de le détériorer par leur puissance thermique. Mais pourquoi ne pas donner un objectif plus ambitieux encore à cette coopération entre société et géologie : des colis «froids et plus sûrs », préparés au cours d'un entreposage actif, pour rendre le stockage d'exploitation plus aisé dans le court terme, et de meilleure protection contre les remontées de radioéléments dans la très longue période?

Or même en se restreignant à un premier niveau d'ambition, la surveillance, la maintenance et le tri des colis pendant la phase d'entreposage, un travail de définition industrielle est nécessaire pour s'entendre sur comment l'Andra opérerait cette fonction, puisque cela devrait être à l'évidence de sa responsabilité (cela n'est pas le cas aujourd'hui et nécessiterait une modification des missions de l'Andra). Aller plus loin, ne pas se contenter de colis rendus «plus sûrs » par surveillance et élimination, cela exige de passer, pendant les deux cents ans où les colis sont gérés en entrepôt, de la maintenance curative à une maintenance prédictive et à des actions correctives. Donc avant tout, cela exige d'inventer les outils d'inspection non intrusive de ces petits réacteurs nucléaires que sont les colis, puis les actions susceptibles de les rendre plus sûrs. N'y a-t-il pas dans les acquis de recherches précédentes ou voisines des bases pour y répondre par des recherches appliquées?

Au total, ouvrir une perspective ambitieuse de collaboration entre société et géologie, entre entreposage actif de longue durée et stockage géologique irréversible, nécessite une recherche préalable de faisabilité et une définition du rôle assigné à chaque phase, voire d'invention d'instruments d'inspections et d'actions industrielles. Cette phase doit et peut bien entendu intégrer naturellement la demande formulée par beaucoup d'experts d'ouvrir des recherches sur la transmutation par laser, manifestement bien reçue par la population. Que 1'on vise d'ailleurs le recours au laser pour diminuer la radioactivité des déchets par transmutation après séparation chimique et rendre les colis définitivement inoffensifs, ou pour constituer un des moyens de les rendre «plus sûrs ». Se donner une vingtaine d'années pour ce faire ne paraît pas excessif.

On ne peut prétendre avoir dans les lignes précédentes tout dit sur ce que devraient être ces recherches au point de convaincre de leur utilité. Pour parvenir à un cahier des charges plus précis et convaincant, dans l'esprit de confiance poursuivi ici, il suffit de puiser dans l'une des meilleures réalisations de l'ouverture à la société de l'IRSN, inaugurée et mise au point par sa fondatrice Annie Sugier, celle d'un «groupe pluriel de 
réflexion », pratiquée avec succès pour les déchets miniers du Poitou ou encore par le groupe radio écologique nord Cotentin (Ministère de l'écologie, 2007). Outre sa contribution à la confiance, l'intérêt substantiel d'un tel format est de tenir ensemble la définition de l'exigence et celle des moyens de l'atteindre, dans un fructueux va-et-vient. Car l'objectif «plus sûrs » est à définir en même temps que les moyens de l'atteindre. Plus sûrs jusqu'à quel point? Faut-il aller jusqu'au niveau d'exigence suédois, viser des colis aussi longtemps et complètement sûrs que s'ils étaient ceinturés de cuivre, au point de pouvoir être stockés dans des galeries de granit où l'eau est présente? Peut-on même espérer les rendre définitivement inoffensifs par transmutation préalable des radionucléides grâce à l'industrialisation des récentes découvertes sur le laser? Ou doit-on chercher plus modestement une coopération dans le temps entre le confinement par le colis, au besoin retravaillé, et le confinement par l'argile, pour assurer les habitants que les remontées de radioéléments seront maîtrisées dans les millénaires?

Résumons: l'ouverture proposée ici est faite d'étapes successives. La première est assignée à un groupe d'expertise plurielle dont l'objectif est programmatique: définir et justifier, en quelques mois, un programme de recherches. Ce programme aurait lui une vingtaine d'années, en temps masqué par rapport à la phase pilote de CIGEO, pour définir opérationnellement les rôles complémentaires de la société, dans un entrepôt à l'échelle centenaire, et de la géologie, par un stockage irréversible à l'échelle millénaire, pour éliminer les peurs de court terme, ce qui paraît atteignable par des mesures organisationnelles, et maîtriser au mieux les risques de remontée de radioéléments à long terme, certainement plus difficile.

\section{Une telle stratégie pour la confiance a toutes raisons, aujourd'hui, de s'imposer}

D'abord et surtout parce que les responsables du nucléaire n'ont plus les raisons de s'y opposer qu'ils avaient en 2006. Ensuite parce que, quoique difficile, cette voie peut, et doit, être engagée par la CNDP comme prolongement et confirmation de sa décision, refondatrice de son indépendance, d'ouvrir le débat sur les alternatives à CIGEO.

Les responsables n'ont plus de raison de s'y opposer en effet. D'abord parce que même une solution définitive pour les déchets à vie longue ne couperait pas l'herbe sous le pied à certaines associations, comme ont pu le penser, au point d'en faire leur doctrine exclusive, les parlementaires les plus impliqués dans le sujet durant plusieurs décennies. Certes, le problème des déchets à vie longue reste un argument contre le nucléaire lui-même. Mais alors qu'il était le seul en 20052006 , à un moment où le baromètre de la confiance dans les centrales était au plus haut, d'où la déclaration de M. Gadonneix, il n'est aujourd'hui ni le seul ni même le principal levier de ceux qui prônent l'arrêt du nucléaire.

Ensuite parce que cette stratégie de la confiance n'arrête pas le projet actuel. Au vu de tous les avis donnés ces derniers temps (IRSN, ASN, collège d'experts internationaux) la décision de procéder à une «phase pilote», si elle est vraiment «pilote», donne au moins vingt ans pour la recherche que suppose cette stratégie. On dispose à nouveau d'un temps masqué durant lequel une recherche parallèle à la phase pilote de CIGEO est possible. Le temps est, comme en 2005-2006, le paramètre principal du problème des déchets nucléaires. Il ne doit plus être instrumentalisé pour donner lieu à une politique du fait accompli, le pire poison de la confiance. Ce devrait être l'apport du débat PNGMDR que de faire la clarté sur le sujet. Avec les éléments dont nous disposons, il nous semble que l'Andra a raison de vouloir démarrer la phase pilote de CIGEO rapidement. Mais il doit s'agir d'une vraie phase pilote, sanctionnée par une évaluation et une décision politique. Dans ces conditions on dispose bien d'un temps masqué pour mettre en œuvre une stratégie inspirée de celle proposée par le débat de 2006.

Enfin parce que, plus fondamentalement, le nucléaire pourrait beaucoup regretter de préférer jouer le triptyque «légitimité-autorité-gendarmerie», ou en termes plus crus «les chiens aboient, la caravane passe». Même si les responsables ont raison de penser que Bures n'est pas Notre-Dame-des-Landes, parce que la démographie n'est pas la même, la population silencieuse leur paraît acquise, et peut-être même parce que les associations nationales opposées au nucléaire pourraient avoir intérêt à coller de moins près aux associations radicales locales qu'elles ne l'ont fait à NotreDame-des-Landes, le risque de gestes de police, anodins pour les uns, inacceptables pour la population, est immense. Ces gestes peuvent détruire la confiance des plus favorables au projet. Certes Bures n'est pas Notre-Dame-des-Landes. Mais Bures pourrait être Sivens.

Mais si cette stratégie pour la confiance de 2005-2006 est adaptable aux réalités sociétales d'aujourd'hui, et si elle n'a plus de raison d'être rejetée par les responsables, elle reste difficile. Car sa définition technique, encore bien floue ici, on doit le reconnaître, nécessite des efforts d'ouverture, d'imagination et d'ingéniosité. Nous avons suggéré un processus sociotechnique pour la préciser, qui a au moins le mérite de la progressivité, et donc en permanence adaptable, voire réversible. Ce processus commence par un groupe d'expertise plurielle de quelques mois, pour définir les recherches à conduire, elles sur une vingtaine d'années, en temps masqué par rapport à CIGEO, afin de spécifier le rôle industriel de la période bi-séculaire de gestion active par la société et les simplifications qu'elles apporteraient au projet CIGEO réversible actuel.

Voie difficile certes, mais pas impossible, au moins pour en faire le premier pas. D'abord parce que les compétences existent, notamment à l'IRSN, pour conduire le groupe d'expertise plurielle initial, grâce à la pratique et à l'engagement, désormais bien reconnus, de ses ingénieurs pour l'ouverture à la société. Mais aussi parce que, grâce à la décision qu'a prise la CNDP d'ouvrir la controverse sur les alternatives à CIGEO au sein du débat PNGMDR, celle-ci dispose d'un instrument qui lui donne le pouvoir et les moyens de lancer ce groupe pluriel programmatique: l'expertise complémentaire inscrite dans la loi de 2002 (Legifrance, 2002), pendant le débat et/ou lors de la concertation d'après débat. Il y va pour cette commission de la confirmation qu'elle est bien décidée à promouvoir une participation «pour un développement durable exigeant» (Mercadal, 2011), certes dotée d'un simple pouvoir d'influence, mais d'une influence que la société civile a du temps et des moyens d'armer d'arguments sérieux. Il semble bien que cette conception de la 
participation comme voie de recherche d'un développement durable exigeant ${ }^{1}$ soit celle de sa Présidente, Mme Chantal Jouanno (Jouanno, 2019). La CNDP ne le doit-elle pas aussi aux experts et associations qui ont joué avec elle le jeu de ce débat PNGMDR?

\section{Références}

Bourguignon M, Bérard P, Bertho JM, Farah J, Mercat C, Comité éditorial de Radioprotection. 2016. Radioprotection : quel avenir? Radioprotection 2017 52(1): 13-20. DOI: 10.1051/radiopro/ 2017007.

Caussade P, Forray N, Michel Massoni M. 2016. Étude sur les alternatives pour le développement aéroportuaire du Grand-Ouest. Rapport $N^{o}$ 010459-01 du Conseil Général de l'Environnement et du Développement Durable.

CNDP. 2006. Mercadal et al. Compte-rendu du débat public sur les options générales en matière de gestion des déchets radioactifs de haute activité et de moyenne activité à vie longue.

CNDP. 2018. Cinquième édition du plan national de gestion des matières et déchets radioactifs. Décision CNDP $N^{\circ} 2018 / 25 /$ PNGMDR/1. Disponible sur https://www.debatpublic.fr/plannational-gestion-matieres-dechets-radioactifs-pngmdr.
CNDP. 2019. Clarification des controverses techniques, Note de synthèse CNDP, Débat Public PNGMDR, 21 mars 2019.

IRSN. 2019a. Panorama international des recherches sur les alternatives au stockage géologique des déchets de haute et moyenne activité à vie longue. Rapport IRSN/2019-00318. Disponible sur https://www.irsn.fr/FR/expertise/rapports exper tise/surete/Pages/Rapport-IRSN-2019.

IRSN. 2019b. Analyse des possibilités d'entreposage à sec de combustibles nucléaires usés de type MOC ou URS. Rapport IRSN/2019-00265. Disponible sur https://www.irsn.fr/FR/exper tise/rapports_expertise/surete/Pages/Rapport-IRSN-2019.

Jouanno C. 2019. La démocratie participative pour une planète meilleure. Interview accordée à France Culture le 9 juin 2019.

Legifrance. 2002. Loi $n^{\circ} 2002-272$ du 27 février 2002 relative à la démocratie de proximité.

Legifrance. 2006a. Loi $n^{\mathrm{o}} 2006-686$ du 13 juin 2006 relative à la transparence et à la sécurité en matière nucléaire.

Legifrance. 2006b. Loi $\mathrm{n}^{\mathrm{o}} 2006-739$ du 28 juin 2006 de programme relative à la gestion durable des matières et déchets radioactifs (modifiée par la loi $\mathrm{n}^{\mathrm{o}}$ 2016-1015 du 25 juillet 2016).

Mercadal G. 2011. Le débat public: pour quel «développement durable? Presse des Ponts.

Ministère de l'écologie. 2007. Le groupe radioécologique Nord Cotentin. L'expertise pluraliste en pratique. La documentation française ed.

Citation de l'article : Mercadal G. 2019. La gestion des déchets radioactifs à vie longue : confiés à l'argile ou bien gérés par la société? Radioprotection 54(3): 169-173

\footnotetext{
${ }^{1}$ Le Président de la République lui-même a utilisé le concept de «développement durable exigeant» dans un discours devant le congrès des collectivités territoriales en février 2018.
} 